\title{
Crystallographic Studies of Molecular Complexes Containing Hexafluorobenzene
}

\author{
Tor Dahl
}

Institute of Mathematical and Physical Sciences, University of Troms $ø$, P.O. Box 953, N-9001 Tromsø, Norway

\begin{abstract}
Dahl, T., 1988. Crystallographic Studies of Molecular Complexes Containing Hexafluorobenzene. - Acta Chem. Scand., Ser. A 42: 1-7.

A survey is made of the crystallographic studies of 1:1 molecular complexes of hexafluorobenzene with aromatic hydrocarbons and aromatic amines. In all complexes investigated, the partner molecules are stacked alternately in infinite columns. Many of the complexes have disordered crystal structures at room temperature, and form twinned crystals with ordered structures on cooling. Clear trends are observed regarding intermolecular distances and molecular overlap. It is, however, difficult to give a theoretical interpretation of the results.
\end{abstract}

\section{Dedicated to Professor Olav Foss on his 70th birthday}

The understanding of the nature of intermolecular forces is essential in chemistry and physics. Quantum mechanical calculations of such forces with satisfactory accuracy are still very difficult, even for small molecules. As the molecular packing in crystals depends on the intermolecular forces, X-ray crystallography is an important experimental method for studying these forces. Information may be obtained in a systematic way by this method when the molecular packing is compared in a series of closely related compounds.

Electron donor-acceptor complexes, also called charge-transfer complexes, are compounds which are well suited for such studies. Investigations of these complexes by various methods have been reviewed by Foster ${ }^{1}$ and Herbstein, ${ }^{2}$ and $\mathrm{X}$-ray crystallographic investigations in particular by Prout and Kamenar. ${ }^{3}$ One important class of electron donor-acceptor complexes, called $n-\sigma^{*}$ complexes, are those between lone-pair donors and halogen-containing acceptors. The crystal structures of these complexes, the majority of which have been determined by Hassel and coworkers, ${ }^{4}$ seem to obey relatively simple rules, both with respect to intermolecular distances and orientation of the molecules.

In $\pi-\pi^{*}$ complexes, where the transfer of charge occurs between $\pi$-orbitals of aromatic or unsaturated aliphatic molecules, the feature com- mon to most of the crystal structures is that the partner molecules are stacked alternately in infinite columns. The overlap of adjacent molecules in the stacks and the interplanar distance between these molecules are, however, difficult to fit into a coherent pattern. In their review article Prout and Kamenar state concerning these structures: "Perhaps the most interesting feature - is the complete lack of any systematic relationship associated with the mean interplanar spacing." The main reason for the absence of trends in these structures is probably that bulky substituents on the benzene rings in most of the complexes are in close contact with neighbouring molecules, both within the stack and in different stacks. The molecular overlap and the interplanar distance are thus influenced greatly by steric factors.

Crystalline 1:1 molecular complexes between hexafluorobenzene (HFB) and aromatic electron donors were first reported in $1960 .{ }^{5}$ From cell dimension and space group determinations of some of these complexes it was concluded in 1965 that the partner molecules are stacked alternately, as in $\pi-\pi^{*}$ complexes. $^{6}$ Spectroscopic studies showed, however, that while the characteristic charge-transfer bands are present in the ultraviolet spectra of the complexes with aromatic amines, no such bands could be observed in the spectra of the complexes with aromatic hydrocarbons. ${ }^{7}$ It has also been shown by various 
experimental methods that HFB probably is a $\sigma^{*}$. rather than a $\pi^{*}$-acceptor ${ }^{8,9}$ The question of the nature of these complexes thus stands as a challenge to many chemists and has been a matter of much dispute. ${ }^{10} \mathrm{~A}$ value of $+1.0(3) \mathrm{kJ} \mathrm{mol}^{-1}$ for the enthalpy of formation of the solid complex between benzene and HFB has been found by calorimetric measurements. ${ }^{11}$ This result and the observation that the crystals of all complexes with HFB are extremely unstable when exposed to the atmosphere at room temperature indicate that the intermolecular forces are weak.

The purpose of the crystal structure determinations of complexes containing HFB has been to elucidate the nature of the intermolecular forces by comparing the molecular packing in a series of closely related complexes. It was considered possible to observe trends by such a comparison if molecules of similar molecular shape and chemical properties were chosen as partners for HFB. For this reason the author has investigated the HFB-complexes with the hydrocarbons $p$-xylene, ${ }^{12}$ mesitylene, ${ }^{13}$ durene ${ }^{14}$ and hexamethylbenzene, ${ }^{15,16}$ and with the amines $N, N$-dimethylaniline, ${ }^{17,18} \quad N, N$-dimethyl-p-toluidine ${ }^{19} \quad$ and $N, N, N^{\prime}, N^{\prime}$-tetramethylphenylenediamine. ${ }^{20}$ In addition, Overell and Pawley ${ }^{21}$ have investigated the complex with deuterobenzene. Structure determinations have also been performed for the complexes between naphthalene and octafluoronaphthalene, ${ }^{22}$ and between biphenyl and perfluorobiphenyl, ${ }^{23}$ which may be regarded as members of the same family of complexes.

For interpretation of the results it was considered relevant to compare the structures of HFB complexes with those of the corresponding fluoranil complexes. Fluoranil has approximately the same molecular shape as HFB, but is a relatively strong $\pi^{*}$-acceptor. ${ }^{1,2} \mathrm{~A}$ comparison of the structures should therefore show differences which are due to chemical rather than steric effects. For this reason, structure determinations of the complexes of fluoranil with durene ${ }^{24}$ and $N, N$-dimethylaniline ${ }^{25}$ have been performed.

\section{Disorder, thermal vibrations and twin formation}

Disorder and large thermal vibrations due to reorientation of the molecules in the crystals have been observed for many electron donor-acceptor complexes, especially those of the $\pi-\pi^{*}$ type. ${ }^{26}$ In complexes containing HFB these features are even more striking than in other classes of complexes. The main causes of this are probably the small size of the F atoms and the disc-shape of the HFB molecule, which allow the molecule to rotate without serious steric hindrance.

For the complexes with deuterobenzene and with hexamethylbenzene the space group at room temperature is $R \overline{3} m$ (or possibly $R 3 m$ ). The investigations of these structures showed that they have a very complicated disorder, and the structures arrived at are very uncertain and will not be discussed in this article. The same space group has also been observed for the HFB complexes of 1,2-dimethylbenzene, 1,2,3,5-tetramethylbenzene, pentamethylbenzene and aniline at room temperature. ${ }^{27} \mathrm{~A}$ similar kind of disorder is probably present in the structure of these complexes, but no attempts at structure determination have been made.

In the complexes of HFB with durene and $N, N, N^{\prime}, N^{\prime}$-tetramethylphenylendiamine, which were investigated at room temperature, more than one orientation of the HFB molecule had to be assumed, and in the room temperature structures of the complexes with $N, N$-dimethylaniline and $N, N$-dimethyl-p-toluidine two orientations of the aromatic amine had to be assumed.

For all complexes investigated at temperatures between room temperature and $-40^{\circ} \mathrm{C}$ the largest $U_{\mathrm{ii}}$-values for the $\mathrm{F}$ atoms are $0.20-0.27$

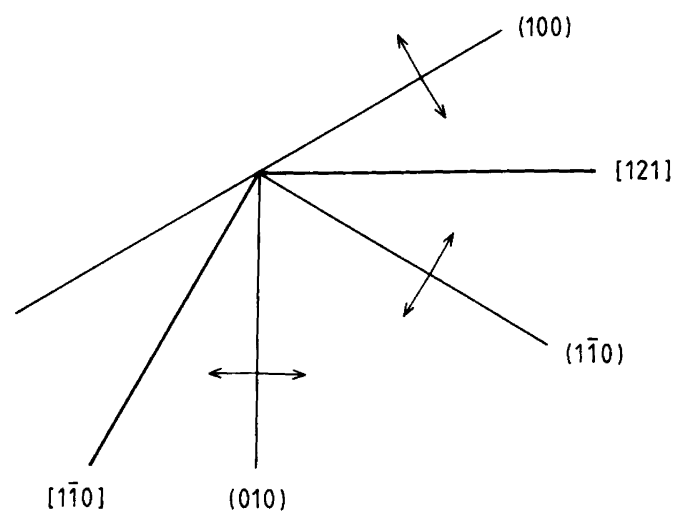

Fig. 1. Pseudo-trigonal axes and non-equivalent pseudo-mirror planes of the triclinic form of the complex between HFB and hexamethylbenzene. The arrows indicate how the crystal may grow in different directions to form repeated twins. 
$\AA^{2}$, and the largest r.m.s. angles of libration of the HFB molecules are $7-12^{\circ}$. These large values may be due not only to thermal vibrations, but may also possibly reflect some orientational disorder. The vibrations of the other partner molecule in most of the complexes are somewhat smaller than those of the HFB molecule.

The disorder and the large thermal vibrations observed in these structures are in agreement with the results of NMR investigations of the crystalline complexes of HFB with benzene, mesitylene and durene. ${ }^{28}$ These results indicate reorientation of both partner molecules at room temperature, and of the HFB molecule even at $77 \mathrm{~K}$.

The crystals of many of the complexes are destroyed on cooling, probably as a result of phase transitions. For other complexes, phase transitions cause formation of twinned crystals of the pseudo-merohedral type ${ }^{29}$ at low temperatures. Mirror planes in the room temperature forms are transformed to pseudo-mirror planes, which are twin planes in the low temperature crystals. The space group $R \overline{3} m$ of the room temperature form of the complex with hexamethylbenzene has three equivalent mirror planes. In the triclinic crystals formed below $0{ }^{\circ} \mathrm{C}$ these planes become non-equivalent pseudo-mirror planes, and the crystals thus have three non-equivalent twin planes (Fig. 1). As a result of this, repeated twins with many individuals are formed. The crystals of the complex with $p$-xylene are triclinic with one pseudo-mirror plane at room temperature. Single crystals of this complex, which is very volatile, are transformed to twinned crystals when kept in sealed capillaries at room temperature for a few hours.

Twinned crystals were used for the determination of the structure of the complex with $p$-xylene and those of the low temperature forms of the complexes with hexamethylbenzene and $N, N$-dimethylaniline. For the two former complexes film technique was used, sets of reflections from the same individuals being picked out visually from the films. For the latter complex diffractometer data were used. In this case steps had to be taken to exclude overlapping reflections from the intensity data.

In the room temperature structures of the complexes with $N, N$-dimethylaniline and $N, N$-dimethyl-p-toluidine the amine is situated on a twofold symmetry axis, and the geometry of the $\mathrm{N}$ atom therefore appears to be planar. However, in the low temperature structure of the $N, N$ dimethylaniline complex the geometry was found to be non-planar, as also observed for uncomplexed $N, N$-dimethylaniline in the gas phase. ${ }^{30}$ The apparent planar geometry in the room temperature structure is thus probably a result of disorder in addition to that already assumed in the determination of the room temperature structure. Similar additional disorder may be present in the structure of the $N, N$-dimethyl-p-toluidine complex.

\section{Interplanar distances}

The mean interplanar distances (Table 1) are relatively large compared to those observed for ordinary $\pi-\pi^{*}$ complexes. This is probably due in

Table 1. Mean interplanar distances. The standard deviations are well below $0.01 \AA$ for all the complexes. In the structures marked with asterisks the two non-equivalent distances on different sides of the molecules are not significantly different.

\begin{tabular}{lll}
\hline Complex & Temp./K & Distance/A \\
\hline p-Xylene - HFB & 293 & 3.55 \\
Mesitylene - HFB* & 238 & 3.56 \\
Durene - HFB & 293 & 3.51 \\
Hexamethylbenzene - HFB & 233 & 3.43 \\
N,N-dimethylaniline - HFB & 293 & 3.52 \\
$N, N$-dimethylaniline - HFB & 120 & 3.39 \\
$N, N$-dimethyl-p-toluidine - HFB & 293 & 3.50 \\
$N, N, N^{\prime}, N^{\prime}$-tetramethylphenylenediamine - HFB & 293 & 3.43 \\
\hline
\end{tabular}


part to the large space required for rotation of the methyl groups, which occurs near room temperature in this kind of complex. ${ }^{28}$ The shortest intermolecular distances to $\mathrm{H}$ atoms during such rotation were calculated for all the structures except the low temperature structure of the $N, N$ dimethylaniline complex: If van der Waals radii of $1.2,1.35$ and $1.7 \AA$ are assumed for the $\mathrm{H}, \mathrm{F}$ and $\mathrm{C}$ atoms, respectively, ${ }^{31}$ such intermolecular

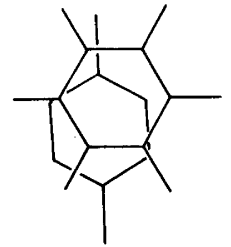

(a)

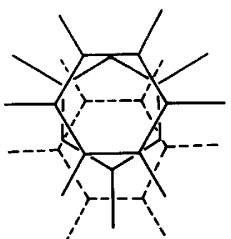

(b)

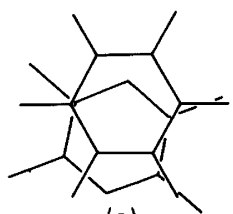

(c)

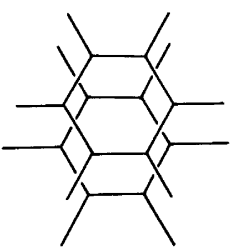

(d)

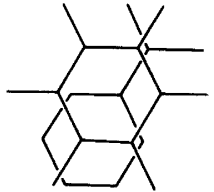

(e)

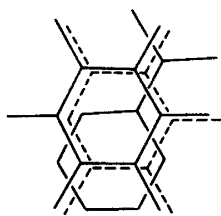

(f)
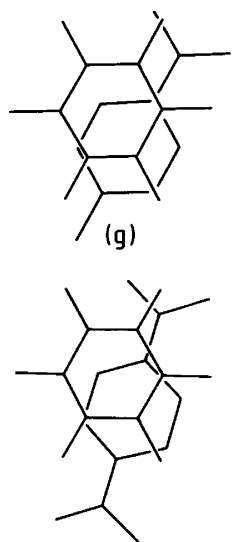

(h)
Fig. 2. Overlap diagrams of the HFB complexes of (a) p-xylene, (b) mesitylene, (c) durene, (d) hexamethylbenzene (triclinic form), (e) $N, N$ dimethylaniline (monoclinic form), (f) $N, N$ dimethylaniline (triclinic form), (g) $N, N$-dimethyl- $p$ toluidine and (h) $N, N, N^{\prime}, N^{\prime}$-tetramethylphenylenediamine. In (b) and (f) the overlap diagrams are different on different sides of the molecules. In (h) only the major orientation of the HFB molecule is shown. In (c), (e) and (g) only one of the two equivalent orientations of the other partner molecule is shown. distances below the van der Waals distances are present only in the complex with hexamethylbenzene, where the shortest $\mathrm{H}-\mathrm{C}$ distance is $2.6 \AA$. No intermolecular distances between non- $\mathrm{H}$ atoms are below the van der Waals distances in any of the complexes.

On comparing the interplanar distances in the complexes with aromatic hydrocarbons with those in the complexes with aromatic amines clear trends are observed. In the complexes with aromatic amines at room temperature the distances decrease with increasing number of amino groups and methyl groups. In the complexes with aromatic hydrocarbons the distances decrease when the number of methyl groups is increased beyond three.

Two of these complexes were investigated below room temperature. If the complex with mesitylene had been investigated at room temperature, the interplanar distance would probably have been larger and the trend would have been even more pronounced. An unusually large increase in interplanar distance with increasing temperature, viz. $6.5 \cdot 10^{-4} \AA \mathrm{K}^{-1}$, has been observed for hexamethylbenzene. ${ }^{32}$ Even in the light of this value and the observed temperature dependence of the interplanar distance in the HFB complex with $N, N$-dimethylaniline (Table 1) it seems unlikely that the shortening of the distance in the complex with hexamethylbenzene relative to that in the complex with durene should be due to the temperature difference alone.

\section{Molecular overlap}

The projections of adjacent molecules onto their average plane, the overlap diagrams, are shown in Fig. 2. The benzene rings are twisted $30^{\circ}$ relative to each other in the complexes with $p$-xylene and mesitylene, $19^{\circ}$ in the complex with durene, while they are parallel in the hexamethylbenzene complex. Increasing the number of methyl groups of the aromatic hydrocarbons beyond three has thus both the effect of reducing the interplanar distances and of altering the relative orientations towards parallelism.

An overlap diagram very similar to that for the hexamethylbenzene complex, with a $\mathrm{C}-\mathrm{C}$ bond approximately above and parallel to a diagonal of the benzene ring of the adjacent molecule, is observed for the complexes with $N, N$-dimethylaniline and $N, N$-dimethyl-p-toluidine. Both these 


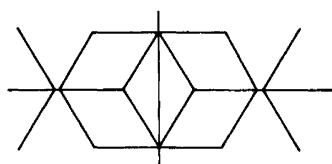

(a)

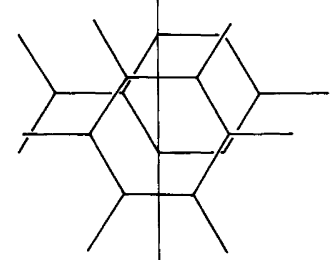

(b)

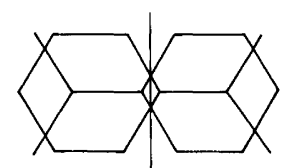

(c)

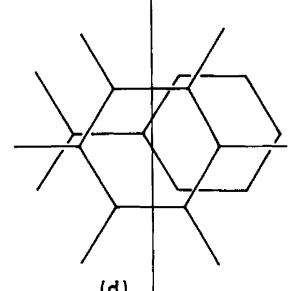

(d)
Fig. 3. The disorder of the aromatic amine and the overlap diagram for the HFB complex with $N, N$ dimethylaniline [(c) and (d)] and with $N, N$-dimethyl-ptoluidine [(a) and (b)]. From Ref. 19.

structures are disordered at room temperature with the amine in two orientations related by a mirror plane. As observed in other disordered structures, the molecules occupy approximately the same space in the two orientations (Fig. 3). For that reason their benzene rings have different positions relative to the mirror plane and thus also relative to the HFB molecule. As a result of different inclinations relative to the stacking axis, one of the $\mathrm{C}-\mathrm{C}$ bonds nearest to the amino group in both complexes is still approximately above, and parallel to, a diagonal of the ring of the hexaflucrobenzene molecule. The overlap diagrams are thus similar in spite of different steric requirements in these two structures. Because of the tetrahedral character of the $\mathrm{N}$ atoms, the steric requirements are different on different sides of the amine in the low temperature structure of the $N, N$-dimethylaniline complex. The two corresponding overlap diagrams are still extremely similar.

It is interesting to note that a somewhat different overlap diagram is observed for the $N, N, N^{\prime}, N^{\prime}$-tetramethylphenylenediamine complex, in which the benzene ring of the HFB molecule in the major and the minor orientation is twisted 13 and $24^{\circ}$, respectively, relative to that of the amine.

\section{Discussion}

In contrast to the observations made with ordinary $\pi-\pi^{*}$ complexes, clear trends regarding both interplanar distances and molecular overlap are found in the complexes of HFB with aromatic hydrocarbons and aromatic amines. These trends demand a theoretical interpretation. An attempt at an interpretation may be based on the model used by Morokuma and Kitaura ${ }^{33}$ who describe the intermolecular interaction energy as the sum of electrostatic, exchange, polarization, chargetransfer and dispersion energy.

An interplanar distance of $3.38 \AA$ has been observed in the complex of durene with fluoranil at room temperature, i.e. $0.13 \AA$ shorter than in the corresponding complex with HFB. The large interplanar distances in the HFB complexes are thus not only due to bulky substituents, but also to weak intermolecular forces. The observed variations in the interplanar distances indicate that these distances are relatively sensitive to variations in the strength of the intermolecular forces. This may to some extent be due to the "soft" nature of the surface formed by the rotating methyl groups of the hydrocarbons and amines.

An increase in the number of methyl groups in aromatic hydrocarbons, and of amino groups and methyl groups in aromatic amines has the effect both of increasing the polarizabilities and decreasing the ionization potentials of the molecules. The decrease in interplanar distances may thus possibly be the result of both increased dispersion and charge-transfer forces.

The small size of the $F$ atoms and the discshape of the HFB molecule, allowing the molecule to rotate with little steric hindrance, are probably the origin of the clear trends observed regarding molecular overlap, since the overlap is relatively unaffected by steric factors. An interesting observation is the similarity of the overlap diagrams of the complexes with hexamethylbenzene, $N, N$-dimethylaniline and $N, N$-dimethyl- $p$ toluidine. Kitaigorodsky ${ }^{34}$ has pointed out that an overlap diagram approximately as observed for these complexes would give the most effective packing of aromatic molecules. It is, however, doubtful whether this is true for molecules with bulky methyl groups as in the present complexes. In particular, it seems improbable that approximately the same overlap diagram should give the most effective packing on different sides of the $\mathrm{N}, \mathrm{N}$-dimethylaniline molecule, where the steric requirements are different because of the tetrahedral geometry of the amino group. In order to elucidate these points, lattice energy calculations 
based on analytical atom-to-atom potentials ${ }^{34}$ should be performed.

If electrostatic interactions were dominant one would expect overlap diagrams with the centres of the benzene rings directly superposed, which is approximately what is observed for the complex between durene and fluoranil. In the complexes with HFB, however, such overlap is observed only on one side of the mesitylene molecule.

Of the remaining kinds of interaction which may explain the observed overlap diagrams only charge-transfer interaction seems to be worth considering. However, there are important pieces of evidence against this explanation, such as the absence of charge-transfer bands in the spectra of the complexes with aromatic hydrocarbons, and the relatively low electron affinity of HFB ${ }^{35}$ Even if the contribution of chargetransfer interactions to the total interaction energy is small, these interactions may, however, have a considerable effect on the molecular overlap if they are favoured significantly by a certain kind of overlap.

According to quantum mechanical calculations based on PPP $\pi$-electron theory, an overlap diagram similar to those observed for the complexes with hexamethylbenzene, $N, N$-dimethylaniline and $N, N$-dimethyl-p-toluidine corresponds to maximum charge-transfer interaction in $\pi-\pi^{*}$ complexes between substituted benzenes. ${ }^{36}$ The calculations show, however, that these interactions are not very sensitive to variations in the relative orientation of the molecules. The relevance of these results to the complexes of HFB, in which $\sigma^{*}$-orbitals are more involved in the interactions, may be rather limited.

In the complex between $N, N$-dimethylaniline and fluoranil large differences were observed in the interplanar distances and the overlap diagrams on different sides of the amine. This indicates that $N, N$-dimethylaniline is an $n$-donor. For the complex between $N, N$-dimethylaniline and HFB the corresponding interplanar distances and overlap diagrams are approximately equal. If charge-transfer interactions have an influence on the molecular packing, $N, N$-dimethylaniline must therefore be a $\pi$-donor in this complex. This is also in accordance with the observation that the tetrahedral geometry of the amino group is more pronounced in the fluoranil complex than in the HFB complex. The reason why the overlap diagram for the HFB complex with $N, N, N^{\prime}, N^{\prime}$-te- tramethylphenylenediamine is different from those of the complexes with the other two amines and hexamethylbenzene may be that $N, N, N^{\prime}, N^{\prime}$ tetramethylphenylenediamine is an $n$-donor.

Although additional crystal structure determinations would be valuable, it is believed that there are now sufficient experimental data to facilitate a more rigorous theoretical approach to the problem of the nature of the intermolecular forces in complexes containing HFB. The observed trends regarding interplanar distances and molecular overlap in the crystal structures may be a good starting point for theoretical work. It is therefore hoped that these crystal structure determinations may contribute to solving this problem and also to a better understanding of the nature of intermolecular forces in general.

\section{References}

1. Foster, R. Organic Charge-transfer Complexes, Academic Press, London and New York 1969.

2. Herbstein, F. H. In: Dunitz, J. O. and Ibers, J. A., Eds., Perspectives in Structural Chemistry, Wiley, New York 1971, Vol. 4, Chap. 3.

3. Prout, C. K. and Kamenar, B. In: Foster, R., Ed., Molecular Complexes, Elek, London 1973, Vol. 1, Chap. 4.

4. Hassel, O. and Rømming, C. Quart. Rev. 16 (1962) 1.

5. Patrick, C. R. and Prosser, G. S. Nature (London) 187 (1960) 1021.

6. Boyens, J. C. A. and Herbstein, F. H. J. Phys. Chem. 69 (1965) 2153.

7. Beaumont, T. G. and Davis, K. M. C. J. Chem. Soc. $B(1967) 1131$.

8. Yim, M. B. and Wood, D. E. J. Am. Chem. Soc. 98 (1976) 2053.

9. Hitchcock, A. P., Fischer, P., Gedanken, A. and Robin, M. B. J. Phys. Chem. 91 (1987) 531.

10. Swinton, F. L. In: Foster, R., Ed., Molecular Complexes, Elek, London 1974, Vol. 2, Chap. 2.

11. Brennan, J. S., Brown, N. M. D. and Swinton, F. L. J. Chem. Soc., Faraday Trans. 1, 70 (1974) 1065.

12. Dahl, T. Acta Chem. Scand., Ser. A 29 (1975) 170.

13. Dahl, T. Acta Chem. Scand. 25 (1971) 1031.

14. Dahl, T. Acta Chem. Scand., Ser. A 29 (1975) 699.

15. Dahl, T. Acta Chem. Scand. 26 (1972) 1569.

16. Dahl, T. Acta Chem. Scand. 27 (1973) 995.

17. Dahl, T. Acta Crystallogr., Sect. B 33 (1977) 3021.

18. Dahl, T. Acta Crystallogr., Sect. C 41 (1985) 931.

19. Dahl, T. Acta Crystallogr., Sect. B37 (1981) 98.

20. Dahl, T. Acta Chem. Scand., Ser. A 33 (1979) 665. 
21. Overell, J. S. W. and Pawley, G. S. Acta Crystallogr., Sect. B 38 (1982) 1966.

22. Potenza, J. and Mastropaolo, D. Acta Crystallogr., Sect. B 31 (1975) 2527.

23. Naae, D. G. Acta Crystallogr., Sect. B35 (1979) 2765.

24. Dahl, T. and Sørensen, B. Acta Chem. Scand., Ser. B39 (1985) 423.

25. Dahl, T. Acta Chem. Scand., Ser. A 35 (1981) 701.

26. Fyfe, C. A. In: Foster, R., Ed., Molecular Complexes, Elek, London 1973, Vol. 1, Chap. 5.

27. Dahl, T. Unpublished results.

28. Gilson, D. F. R. and McDowell, C. A. Can. J. Chem. 44 (1966) 945.

29. Cahn, R. W. Adv. Phys. 3 (1954) 363.

30. Cervelatti, R., Dal Borgo, A. and Lister, D. G. $J$. Mol. Struct. 78 (1982) 161.
31. Pauling, L. Nature of the Chemical Bond, 3rd ed., Cornell University Press, New York 1960.

32. Woodward, I. Acta Crystallogr. 11 (1958) 441.

33. Morokuma, K. and Kitaura, K. In: Ratajczak, H. and Orville-Thomas, W. J., Eds., Molecular Interactions, Wiley, New York 1980, Vol. 1, Chap. 2.

34. Kitaigorodsky, A. J. Molecular Crystals and Molecules, Academic Press, New York and London 1973.

35. Wentworth, W. E., Limero, T. and Chen, E. C. M. J. Phys. Chem. 91 (1987) 241.

36. Mayoh, B. and Prout, C. K. J. Chem. Soc., Faraday Trans. 2, 68 (1972) 1072.

Received May 4, 1987. 